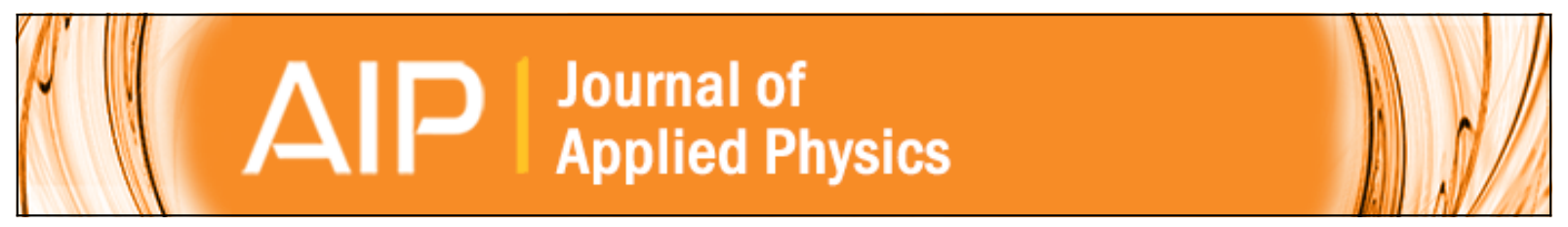

\title{
Raman and electron microscopy analysis of carbon nanotubes exposed to high power laser irradiance
}

Krishna Ramadurai, Christopher L. Cromer, Anne C. Dillon, Roop L. Mahajan, and John H. Lehman

Citation: Journal of Applied Physics 105, 093106 (2009); doi: 10.1063/1.3116165

View online: http://dx.doi.org/10.1063/1.3116165

View Table of Contents: http://scitation.aip.org/content/aip/journal/jap/105/9?ver=pdfcov

Published by the AIP Publishing

\section{Articles you may be interested in}

Ultraviolet pulsed laser irradiation of multi-walled carbon nanotubes in nitrogen atmosphere

J. Appl. Phys. 115, 093501 (2014); 10.1063/1.4864776

Laser irradiation of carbon nanotube films: Effects and heat dissipation probed by Raman spectroscopy

J. Appl. Phys. 114, 024904 (2013); 10.1063/1.4813485

Single-walled carbon nanotubes and nanocrystalline graphene reduce beam-induced movements in highresolution electron cryo-microscopy of ice-embedded biological samples

Appl. Phys. Lett. 99, 133701 (2011); 10.1063/1.3645010

Systematic investigation of sustained laser-induced incandescence in carbon nanotubes

J. Appl. Phys. 107, 064319 (2010); 10.1063/1.3359681

Phonon control in metallic carbon nanotubes due to laser-induced defects

Appl. Phys. Lett. 93, 133102 (2008); 10.1063/1.2990659

\section{MIT LINCOLN} LABORATORY CAREERS

Discover the satisfaction of innovation and service to the nation
- Space Control

- Air \& Missile Defense

- Communications Systems \& Cyber Security

- Intelligence, Surveillance and

Reconnaissance Systems

$$
\begin{aligned}
& \text { - Advanced } \\
& \text { Electronics } \\
& \text { - Tactical Systems } \\
& \text { - Homeland } \\
& \text { Protection } \\
& \text { - Air Traffic Control }
\end{aligned}
$$

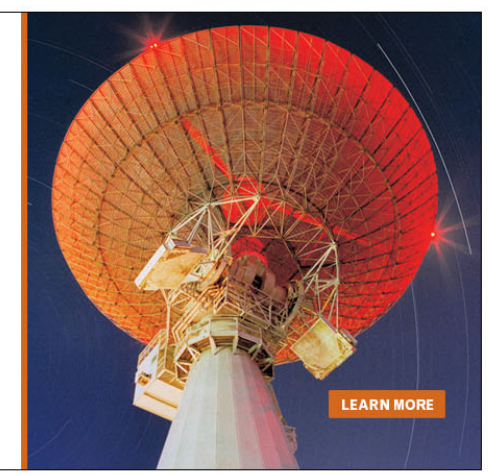




\title{
Raman and electron microscopy analysis of carbon nanotubes exposed to high power laser irradiance
}

\author{
Krishna Ramadurai, ${ }^{1, a)}$ Christopher L. Cromer, ${ }^{2}$ Anne C. Dillon, ${ }^{3}$ Roop L. Mahajan, ${ }^{4}$ and \\ John H. Lehman ${ }^{2}$ \\ ${ }^{1}$ Department of Mechanical Engineering, University of Colorado, Boulder, Colorado 80309, USA \\ ${ }^{2}$ National Institute of Standards and Technology, Boulder, Colorado 80305, USA \\ ${ }^{3}$ National Renewable Energy Laboratory, Golden, Colorado 80305, USA \\ ${ }^{4}$ Department of Engineering Science and Mechanics and Department of Mechanical Engineering, ICTAS \\ (Institute for Critical Technology and Applied Science), Virginia Tech, Blacksburg, Virginia 24060, \\ USA
}

(Received 6 January 2009; accepted 5 March 2009; published online 5 May 2009)

High power laser radiometry requires efficient and damage-resistant detectors. The current study explores the evolving nature of carbon nanotube coatings for such detectors upon their exposure to incrementally increasing laser power levels. Electron microscopy images along with the $D$-band to $G$-band intensity ratios from the Raman spectra from eight irradiance levels are used to evaluate changes before and after the exposure. Electron microscopy images of the exposed multiwalled carbon nanotubes revealed the formation of intermittent pockets of moundlike structures at high power densities exceeding $11 \mathrm{~kW} / \mathrm{cm}^{2}$. Raman spectroscopy measurements also demonstrated higher values for the ratio of the $D$-band intensity to that of the $G$-band, suggesting the possible transformation of nanotubes into structurally different forms of carbon. Exposure to a sample of single-walled nanotubes did not demonstrate the evolution of structural changes, which could be due in part to the higher irradiance levels relative to the damage threshold, employed in the experiment. (C) 2009 American Institute of Physics. [DOI: 10.1063/1.3116165]

\section{INTRODUCTION}

The calibration of high power lasers is often accomplished by the use of water-cooled calorimeters, ${ }^{1}$ which are often comprised of a radiation absorbing cavity and a black coating. Several black coatings have been used ${ }^{2-5}$ to cater to the increasing need of high irradiance lasers. The application of multiwalled carbon nanotubes (MWCNTs) and singlewalled carbon nanotubes (SWCNTs) as thermal detector coatings has also been explored. ${ }^{6-8}$ In our earlier work, we demonstrated a high damage threshold up to $15 \mathrm{~kW} / \mathrm{cm}^{2}$ upon exposure to $1.06 \mu \mathrm{m}$ irradiance for MWCNT coatings. Raman spectra data have suggested the onset of structural changes in the nanotube coating upon exposure to high power irradiance. Other past experiments and simulations also indicated changes in the nanotube samples after exposure to lasers operating at different wavelengths. Bai et al. ${ }^{9}$ grew aligned CNT films on quartz by the use of a chemical vapor deposition process and exposed the film to a $1.06 \mu \mathrm{m}$ neodymium doped yttrium aluminum garnet (Nd:YAG) continuous laser in an atmosphere of carbon dioxide. They observed that the exposed nanotube sample had relatively lesser amorphous carbon content than the unexposed CNTs. The authors determined an optimum irradiance of $19 \mathrm{~W} / \mathrm{cm}^{2}$ for the removal of amorphous carbon. Huang et al. ${ }^{10}$ demonstrated preferential destruction of metallic SWCNTs by exposure to a $514.5 \mathrm{~nm}$ argon ion laser and a $632.8 \mathrm{~nm}$ helium-neon laser. The relative intensities of the Raman signal for the radial breathing modes as well as the $D$-band and $G$-band intensities were used as indicators of the

${ }^{a)}$ Electronic mail: krishna.ramadura@gmail.com. presence of semiconducting and metallic nanotubes in the exposed sample. More recently, Zhang et al. ${ }^{11}$ demonstrated structural changes in double-walled CNTs upon exposure to a $355 \mathrm{~nm}$ pulsed ultraviolet laser. The exposed sample consisted of hollow and solid carbon nanofibers as well as onionlike structures. The authors suggested a transformation mechanism for these carbon structures based on the photon energy, which breaks the structure resulting in the formation of many fragments. By increasing the laser energy, these structures coalesce, eventually leading to the formation of nanoparticles. Hurst et al. ${ }^{12}$ also demonstrated structural and topological changes in nanotube coatings after their exposure to $248 \mathrm{~nm}$ irradiance.

The present work explores the structural evolution of nanotube coatings upon exposure to incrementally higher power levels of a $1.25 \mathrm{~kW} \mathrm{Nd}$ :YAG laser operating at $1.06 \mu \mathrm{m}$. The samples were produced by airbrush spraying of commercially obtained MWCNTs and SWCNTs on copper. The sprayed nanotubes were then selectively exposed to incremental laser irradiance up to the level where visual damage to the coating was observed in the form of continuous sparking. ${ }^{8}$ Selective exposure of the sample to different irradiance levels was then correlated with the structural changes occurring in the coating through electron microscopy and Raman spectroscopy. In addition to the sprayed CNT samples, a commercially obtained vertically aligned MWCNT sample was also tested and analyzed. The topological study of the exposed nanotube coating is motivated by the need for improvements in the nanotube coating for high power laser calibrations. The topology changes could be 
implemented to further improve the damage threshold to higher irradiance levels that will be required of future thermal detectors.

\section{MATERIALS AND METHODS}

Commercially obtained MWCNTs and SWCNTs were used to create the coatings for the experiments. The nanotubes $(10$ wt \%) were dispersed in chloroform and the mixture was sonicated for a duration of $10 \mathrm{~min}$. The dispersed CNTs were sprayed onto a copper substrate and dried in air prior to experiments. The entire sample was divided into a grid of 14 areas (each area was $1 \times 1 \mathrm{~cm}^{2}$ ) with each area exposed to a different irradiance. During irradiance the sample was mounted on a holder with port connections for cooling water. ${ }^{13}$ It is to be noted here that the coating thickness was not uniform over the sample substrate area (62 $\times 24 \mathrm{~mm}^{2}$ ).

The experimental setup employed a $1.25 \mathrm{~kW} \mathrm{Nd:YAG}$ laser operating at $1.06 \mu \mathrm{m}$. The incident radiation was allowed to pass through a beamsplitter. One beam was directed to a separate monitor power meter, while the other beam was focused onto the sample by an aperture and focusing lens arrangement. The sample holder comprised of a rectangular plastic (polyoxymethylene) block with a recess for holding the sample. Ports on either end of the block allowed for the flow of water to cool the sample. The resulting water flow direction was perpendicular to the optical axis, and its mass flow rate was determined by a Coriolis flow meter. Calibrated commercial thermistors were mounted on the inlet and outlet water channels to measure the change in water temperature upon transit through the sample. ${ }^{13}$ The absorbed power was calculated from the cooling water flow rate and the change in the water temperature. The calibration factor used to determine the incident power was evaluated by replacing the sample holder with a water-cooled power meter. Each area on the grid was sequentially exposed to laser light for a fixed duration and increments of irradiance. Then the beam was refocused on a different area on the grid at lower irradiance prior to exposure to the next higher irradiance (relative to the previous area). The procedure was repeated until the irradiance was reached at which continuous sparking on the nanotube sample was observed. One of the areas was left unexposed for comparison with the exposed regions of the nanotube coating.

The grids were then analyzed by the use of Raman spectroscopy and electron microscopy. The Raman spectroscopy measurements were performed by the use of a $488 \mathrm{~nm}$ excitation produced by an argon ion laser operating at $\sim 12 \mathrm{~mW}$. The beam was focused on an area of $\sim 0.25 \mathrm{~mm}^{2}$, and the scattered light was analyzed by the use of a $270 \mathrm{~mm}$ grating spectrometer. ${ }^{8,12}$ For the electron microscopy analysis, the copper substrate was directly attached to the electron microscope sample holder by using carbon tape. Information from the Raman spectra was tabulated to assist in correlation with the structural changes observed by the use of electron microscopy. Further details of the experimental setup are described elsewhere. ${ }^{8,12,13}$ Figures 1(A) and 1(B) show images of the exposed grids of SWCNTs and MWCNTs, respectively.

\section{RESULTS AND DISCUSSION}

An evaluation was performed by Raman spectroscopy on the unexposed and exposed regions of the SWCNT as well as the MWCNT grids. The " $G$-band" is representative of the in-plane vibrational modes of carbon in the curved graphite lattice, whereas the " $D$-band" is generated through the presence of defects and non-nanotube impurities. ${ }^{14,15}$ Figures 2(A)-2(H) depict the Raman spectra for the MWCNT coating upon exposure to different incident power densities. It can be observed that for the sample exposed to $4.94 \mathrm{~kW} / \mathrm{cm}^{2}$ irradiance, a shoulder feature appears in the $G$-band, centered at a Raman shift of $1600-1650 \mathrm{~cm}^{-1}$. The shoulder is seen at higher irradiance as well. There is also evidence of the seed of another peak, centered at a Raman shift of 600-700 $\mathrm{cm}^{-1}$, in the grid area exposed to an irradiance of $10.9 \mathrm{~kW} / \mathrm{cm}^{2}$. The Raman spectra depict the presence of additional peaks at an irradiance of $14.2 \mathrm{~kW} / \mathrm{cm}^{2}$. The exposed copper could have resulted from the locally lower coating thickness resulting in coating ablation at a relatively lower irradiance compared to the damage threshold. The ratios of intensities of the $D$-band and $G$-band $\left(I_{D} / I_{G}\right)$ (see Table I) are always higher for the exposed areas relative to those of the unexposed spot. This result is a representative of changes in the amount of defects and the degree of graphitization in the CNT coating. ${ }^{14}$ Table I also lists the evaluated full width at half maximum (FWHM) of the $D$-band. It can be observed that the FWHM of the $D$-band is consistently lower for the exposed areas compared to that for the unexposed areas, suggesting the removal of amorphous
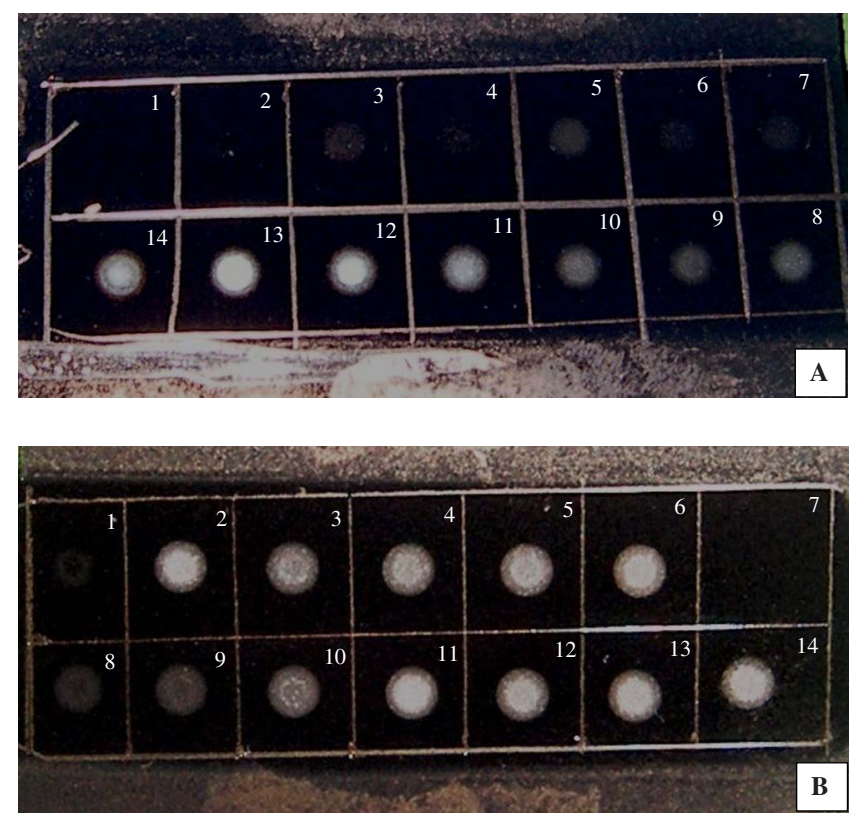

FIG. 1. (Color online) Images of the irradiated (A) MWCNT and (B) SWCNT samples. Both samples were divided into 14 grids. The exposed region is depicted by the bright band at the center of each area. Area 1 was unexposed in the MWCNT sample, while area 7 was unexposed in the SWCNT sample. The other area numbers were assigned with higher values representing exposure at higher irradiance. 

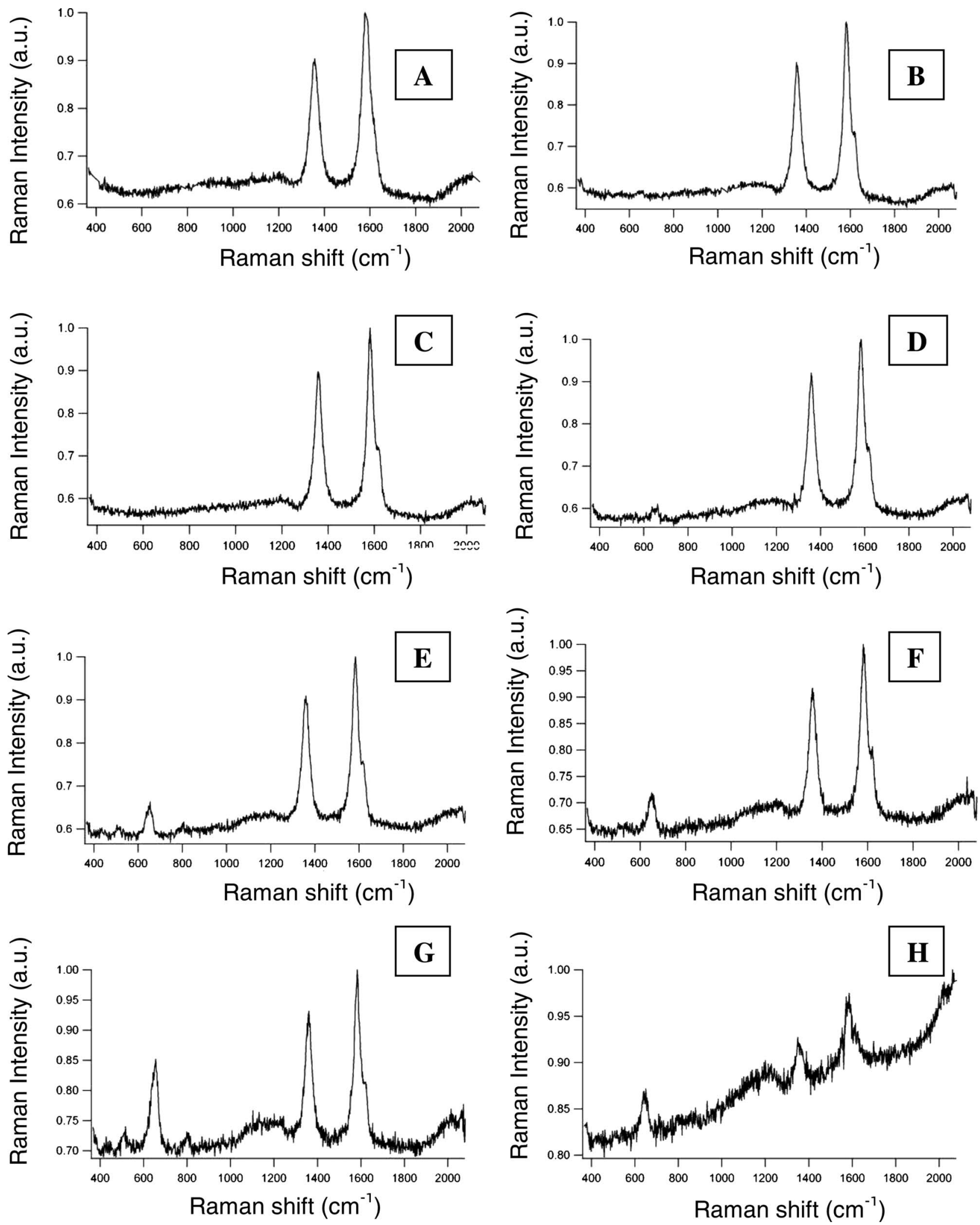

FIG. 2. Raman spectra, normalized to the intensity of the $G$-band of the MWCNT sample exposed to laser irradiance at (A) 0 kW/ $\mathrm{cm}^{2}$ (unexposed), (B) $4.9 \mathrm{~kW} / \mathrm{cm}^{2}$, (C) $8.4 \mathrm{~kW} / \mathrm{cm}^{2}$, (D) $10.9 \mathrm{~kW} / \mathrm{cm}^{2}$, (E) $11.5 \mathrm{~kW} / \mathrm{cm}^{2}$, (F) $13.4 \mathrm{~kW} / \mathrm{cm}^{2}$, (G) $14.2 \mathrm{~kW} / \mathrm{cm}^{2}$, and $(\mathrm{H}) 14.8 \mathrm{~kW} / \mathrm{cm}^{2}$, respectively.

carbon and, possibly, other non-nanotube impurities as well. At the highest irradiance, of $14.8 \mathrm{~kW} / \mathrm{cm}^{2}$, the Raman spectrum is strongly distorted, implying the transformation of CNTs into different carbon structures. There is no general trend in either the FWHM of the $D$-band or the $I_{D} / I_{G}$ ratios as a function of irradiance.
The Raman spectra results were supplemented with scanning electron microscopy (SEM) images of the exposed MWCNT grids. Figures $3(\mathrm{~A})-3(\mathrm{H})$ are SEM images of the exposed MWCNT sample at various incident irradiance levels. The SEM images at incident irradiance exceeding $4 \mathrm{~kW} / \mathrm{cm}^{2}$ depicted bright spots present in the sample. Hung 
TABLE I. Variation in the $I_{D} / I_{G}$ ratio and the FWHM of the $D$-band as a function of incident irradiance for the MWCNT sample.

\begin{tabular}{lcc}
\hline \hline $\begin{array}{l}\text { Irradiance } \\
\left(\mathrm{kW} / \mathrm{cm}^{2}\right)\end{array}$ & $\begin{array}{c}\text { FWHM of } D \text {-band } \\
\left(\mathrm{cm}^{-1}\right)\end{array}$ & $I_{D} / I_{G}$ \\
\hline Unexposed & 44.84 & 0.714 \\
2.5 & 39.04 & 1.057 \\
4.9 & 38.64 & 1.160 \\
6.6 & 37.54 & 1.236 \\
7.8 & 36.10 & 1.061 \\
8.4 & 35.82 & 1.134 \\
9.1 & 43.18 & 1.024 \\
10.9 & 36.34 & 1.059 \\
11.6 & 36.20 & 1.101 \\
12.2 & 33.19 & 0.973 \\
13.4 & 35.94 & 1.093 \\
14.2 & 33.27 & 1.096 \\
14.8 & 34.78 & 1.079 \\
\hline \hline
\end{tabular}

et $a l .{ }^{16}$ also described the presence of bright spots on MWCNT samples exposed to laser irradiation. These spots were attributed to the aggregation of the nanotube bundles upon burnout of selective nanotubes in their three-
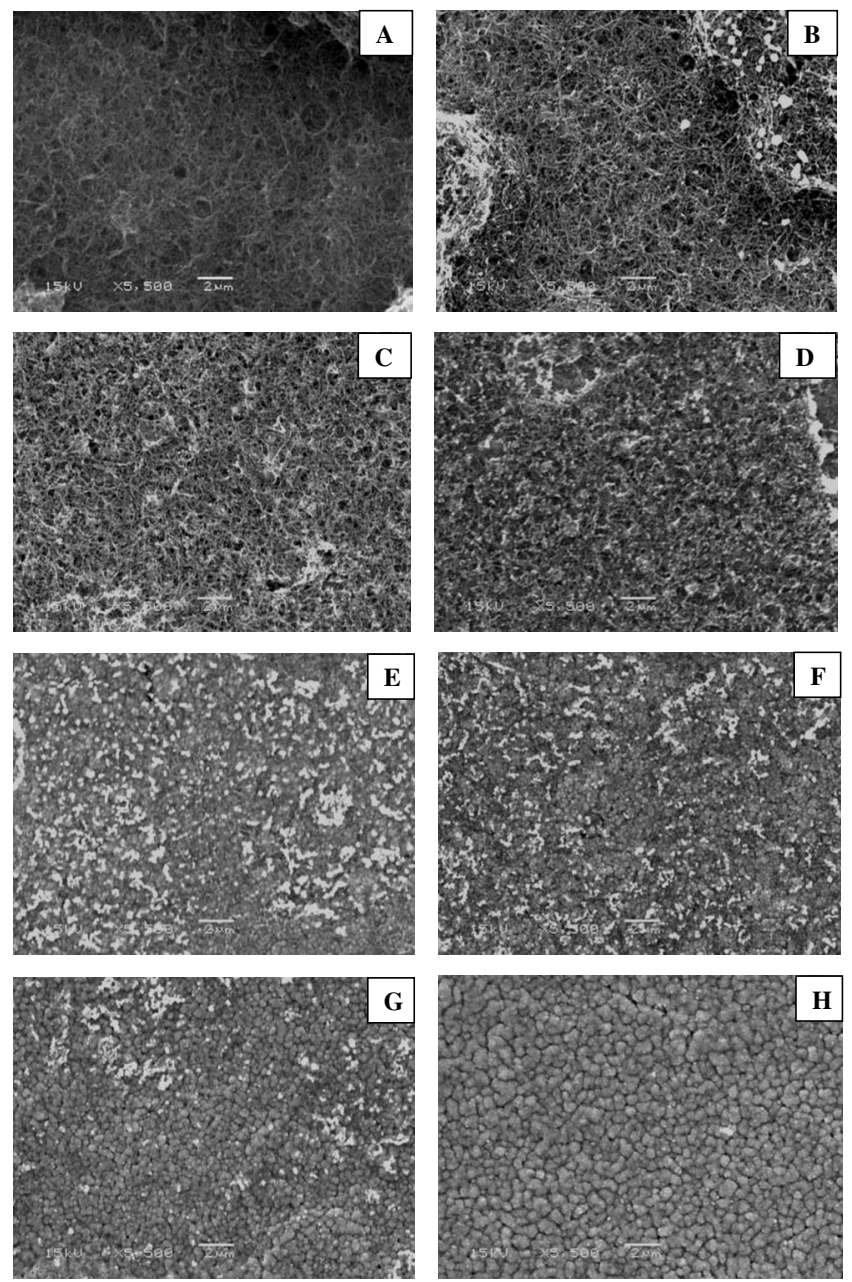

FIG. 3. SEM images of the MWCNT sample exposed to an irradiance of (A) $0 \mathrm{~kW} / \mathrm{cm}^{2}$ (unexposed), (B) $4.9 \mathrm{~kW} / \mathrm{cm}^{2}$, (C) $8.4 \mathrm{~kW} / \mathrm{cm}^{2}$, (D) $10.9 \mathrm{~kW} / \mathrm{cm}^{2}$, (E) $11.5 \mathrm{~kW} / \mathrm{cm}^{2}$, (F) $13.4 \mathrm{~kW} / \mathrm{cm}^{2}$, (G) $14.2 \mathrm{~kW} / \mathrm{cm}^{2}$, and $(\mathrm{H}) 14.8 \mathrm{~kW} / \mathrm{cm}^{2}$, respectively. dimensional prototyping process. In our work, we also attributed the bright spots to aggregates of nanotubes resulting from exposure to high irradiance. There was stronger presence of these aggregates with increasing irradiance levels. At irradiance exceeding $11 \mathrm{~kW} / \mathrm{cm}^{2}$, the SEM images depicted the formation of pockets of nontubular structures on the surface of copper and also suggested their evolution with increasing irradiance. These moundlike structures ranged in diameter from less than 250 up to $500 \mathrm{~nm}$. Similar carbon clusters and protrusions were observed by Kichambare et $a l .{ }^{17}$ in their experiments with exposing CNT coatings to 266 and $1064 \mathrm{~nm}$ pulsed lasers. The results support the claim that the structure of the nanotubes was undergoing changes with increasing irradiance. The higher $I_{D} / I_{G}$ ratios at all the exposures suggested disorder in the nanotube wall. At lower irradiance, the SEM images did not reveal significant changes except for the appearance of bright spots attributed to the bundling of nanotubes. However, at irradiance over $11 \mathrm{~kW} / \mathrm{cm}^{2}$, the excess energy transformed CNTs into carbon nanoparticle aggregates. These aggregates were structurally different from nanotubes as observed in the SEM images and also the Raman data. These nanoparticle aggregates could have eventually coalesced at higher irradiance, to result in the observed carbon clusters. With increasing irradiance, the temperature across the exposed grid area also increased to values sufficient to locally oxidize thin regions of the CNT coating. A similar observation was also made by Zhang et al. ${ }^{11}$ at ultraviolet wavelengths. The carbon clusters so formed at high irradiance, exceeding $14 \mathrm{~kW} / \mathrm{cm}^{2}$, also demonstrated high absorbance efficiencies, as noted in our previous experiments with MWCNT coatings. ${ }^{8}$

In a separate experiment, commercially obtained vertically aligned MWCNTs grown on copper were exposed to irradiance under identical experimental conditions. However, the sample demonstrated a very low damage threshold of $0.5 \mathrm{~kW} / \mathrm{cm}^{2}$. The aligned MWCNTs were grown on the copper substrate by use of a buffer layer to prevent the catalyst nucleating spots from diffusing into the copper. It is possible that upon exposure to the laser irradiance, the thermal energy encountered additional resistance from the buffer layers. The increased resistance led to a high temperature gradient across the MWCNT sample, thereby resulting in lower damage thresholds. The SEM images of the exposed aligned MWCNT sample are shown in Figs. 4(A)-4(D). It is interesting to note that there is aggregation of the nanotube tips at several locations. Localized coating ablation could have resulted in mechanical instabilities in the nanotube bundles, causing individual nanotubes to twist, and while in proximity, strongly bundle by virtue of van der Waals forces.

The experiment was repeated with a SWCNT coating but at lower irradiance compared to that of the MWCNT exposure levels. Figures 5(A)-5(C) depict the Raman spectra of the exposed areas up to irradiance levels of $4.8 \mathrm{~kW} / \mathrm{cm}^{2}$. As was the case with the MWCNT sample, the $I_{D} / I_{G}$ ratio of the exposed samples was higher compared to the unexposed sample (see Table II), suggesting the onset of disorder in the CNT coating. There was no specific trend in the FWHM of the $D$-band evaluated for the different exposed grids. The Raman spectra also depicted spectral peaks at Raman shifts 

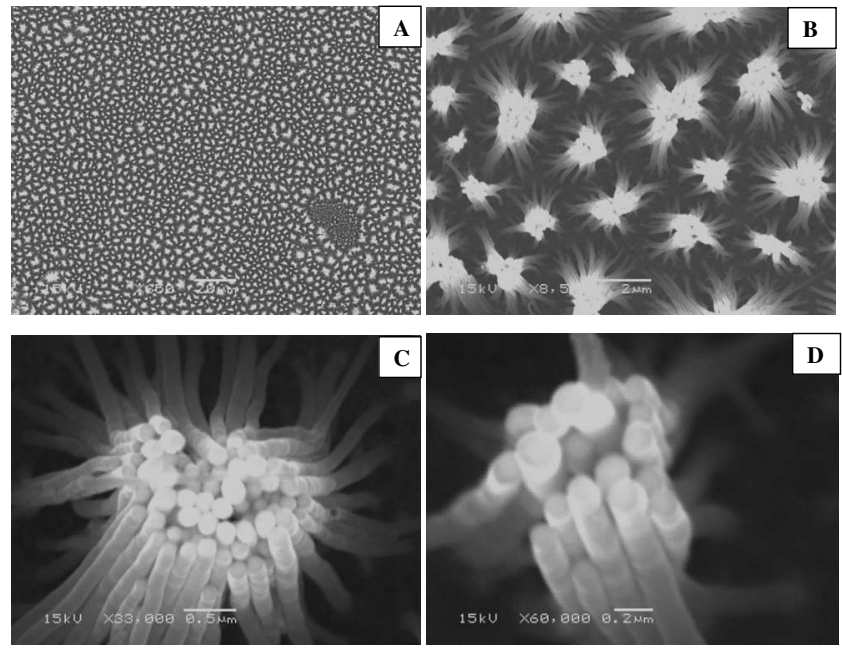

FIG. 4. Progressively increasing magnification SEM images of the vertically aligned MWCNT sample exposed to irradiance at $1.06 \mu \mathrm{m}$.
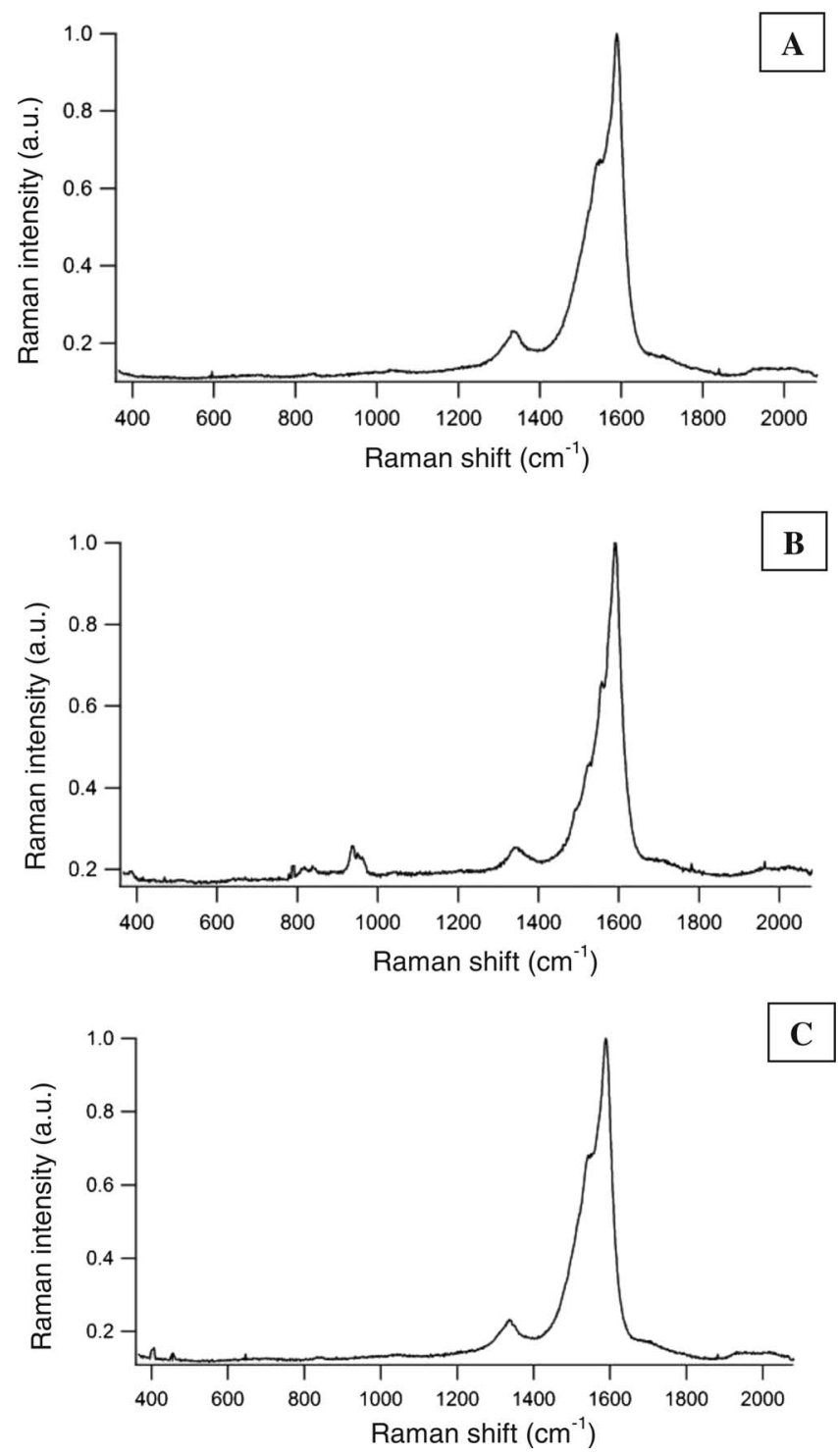

FIG. 5. Raman spectra, normalized to the intensity of the $G$-band of the SWCNT sample exposed to laser irradiance at (A) $0 \mathrm{~kW} / \mathrm{cm}^{2}$ (unexposed), (B) $4.6 \mathrm{~kW} / \mathrm{cm}^{2}$, and (C) $4.8 \mathrm{~kW} / \mathrm{cm}^{2}$, respectively.
TABLE II. Variation in the $I_{D} / I_{G}$ ratio and the FWHM of the $D$-band as a function of incident irradiance for the SWCNT sample.

\begin{tabular}{lcl}
\hline \hline $\begin{array}{l}\text { Irradiance } \\
\left(\mathrm{kW} / \mathrm{cm}^{2}\right)\end{array}$ & $\begin{array}{c}\text { FWHM of } D \text {-band } \\
\left(\mathrm{cm}^{-1}\right)\end{array}$ & $I_{D} / I_{C}$ \\
\hline Unexposed & 28.76 & 0.387 \\
0.5 & 23.81 & 0.389 \\
4.6 & 28.85 & 0.537 \\
4.7 & 29.31 & 0.51 \\
4.8 & 30.43 & 0.408 \\
4.9 & 32.36 & 0.433 \\
\hline \hline
\end{tabular}

ranging from 800 to $1000 \mathrm{~cm}^{-1}$ for the grid area exposed to irradiance around $4.5 \mathrm{~kW} / \mathrm{cm}^{2}$ [see Fig. 5(B)]. However, the high energy shoulder observed with MWCNTs was absent in the case of SWCNTs. SEM images of the SWCNT sample revealed no significant differences from the unexposed sample. The lack of precise control over the incremental steps of the irradiance resulted in exposure levels well over the damage threshold of SWCNT coatings. The high irradiance resulted in the burnout of the SWCNT coating, thereby preventing a detailed study of the structural evolution of the SWCNT coating. However, from the few areas salvaged from complete burnout, there was no evidence of distortion of the Raman spectra or the formation of carbon mounds. Our earlier thermal measurements with nanotube coatings ${ }^{8}$ have demonstrated higher thermal resistance for SWCNTs compared to that for MWCNTs. The higher resistance led to high temperature gradients across the coating even for smaller incident power increments, leading to instantaneous oxidation of the SWCNTs.

\section{CONCLUSIONS}

In this work, a systematic study was undertaken to study the evolution of damage in MWCNT and SWCNT samples exposed to a $1.25 \mathrm{~kW} \mathrm{Nd:YAG} \mathrm{laser} \mathrm{operating} \mathrm{at} \mathrm{a} \mathrm{wave-}$ length of $1.06 \mu \mathrm{m}$. Raman spectroscopy measurements of both damaged samples demonstrated an increase in the $I_{D} / I_{G}$ ratio, which indicates disorder in the nanotube wall. The $G$-band in the exposed MWCNT sample demonstrated the consistent presence of a high energy shoulder centered at $1600-1625 \mathrm{~cm}^{-1}$, which further supported the onset of defects in the MWCNT sample. The FHWM of the $D$-band for the exposed areas in the MWCNT sample was also consistent with the removal of amorphous carbon upon comparison with the unexposed grid area. SEM images revealed the formation of carbon clusters, which resulted from the transformation of the tubular structures at high incident power densities. However, such features were not observed with the exposed SWCNT sample, which had significantly lower damage threshold compared to that for the MWCNT coating. Vertically aligned MWCNTs surprisingly demonstrated a low damage threshold of $0.5 \mathrm{~kW} / \mathrm{cm}^{2}$, which was attributed to the CNT burnout due to increased thermal resistance resulting from the buffer layer between the CNTs and the copper substrate. SEM images of the exposed regions revealed fusion of the nanotube tips. These observations, which depict the structural changes in the MWCNT coating upon exposure 
to high irradiance, have important implications for thermal detector coatings used in high power laser calibrations.

\section{ACKNOWLEDGMENTS}

We thank the CU-NIST seed grant program for providing financial support to this work. We also thank Dr. Xiaoyu Li (NIST, Boulder, CO) for his assistance with the experimental setup and Dr. Katie Hurst for her suggestions on the presented material.

${ }^{1}$ S. R. Gunn, J. Phys. E 6, 105 (1973).

${ }^{2}$ D. B. Betts, F. J. J. Clarke, L. J. Cox, and J. A. Larkin, J. Phys. E 18, 689 (1985).

${ }^{3}$ D. J. Advena, V. T. Bly, and J. T. Cox, Appl. Opt. 32, 1136 (1993).

${ }^{4}$ N. Nelms and J. Dowson, Sens. Actuators, A 120, 403 (2005).

${ }^{5}$ J. H. Lehman, J. E. Theocharous, G. Eppeldauer, and C. Panell, Meas. Sci. Technol. 14, 916 (2003).

${ }^{6}$ J. H. Lehman, C. Engtrakul, T. Gennett, and A. C. Dillon, Appl. Opt. 44, 483 (2005)
${ }^{7}$ E. Theocharous, R. Deshpande, A. C. Dillon, and J. Lehman, Appl. Opt. 45, 1093 (2006).

${ }^{8}$ K. Ramadurai, C. L. Cromer, L. A. Lewis, K. E. Hurst, A. C. Dillon, R. L. Mahajan, and J. H. Lehman, J. Appl. Phys. 103, 013103 (2008).

${ }^{9}$ X. Bai, D. Li, D. Du, H. Zhang, L. Chen, and J. Liang, Carbon 42, 2125 (2004).

${ }^{10}$ H. Huang, R. Maruyama, K. Noda, H. Kajiura, and K. Kadono, J. Phys. Chem. B 110, 7316 (2006).

${ }^{11}$ Y. Zhang, T. Gong, J. Wei, W. Liu, K. Wang, and D. Wu, J. Phys. Chem. C 111, 2901 (2007).

${ }^{12}$ K. E. Hurst, A. C. Dillon, S. Yang, and J. H. Lehman, J. Phys. Chem. C 112(42), 16296 (2008).

${ }^{13}$ K. Ramadurai, C. L. Cromer, X. Li, R. L. Mahajan, and J. H. Lehman, Appl. Opt. 46, 8268 (2007).

${ }^{14}$ A. C. Dillon, A. H. Mahan, P. A. Parilla, J. L. Alleman, M. J. Heben, K. M. Jones, and K. E. H. Gilbert, Nano Lett. 3, 1425 (2003).

${ }^{15}$ A. C. Dillon, M. Yudasaka, and M. S. Dresselhaus, J. Nanosci. Nanotechnol. 4, 691 (2004).

${ }^{16}$ W. H. Hung, R. Kumar, A. Bushmaker, S. B. Cronin, and M. J. Bronikowski, Appl. Phys. Lett. 91, 093121 (2007).

${ }^{17}$ P. D. Kichambare, L. C. Chen, C. T. Wang, K. J. Ma, C. T. Wu, and K. H. Chen, Mater. Chem. Phys. 72, 218 (2001). 